\title{
BMJ Open Cost-effectiveness of new MDR-TB regimens: study protocol for the TB- PRACTECAL economic evaluation substudy
}

\author{
Sedona Sweeney (D) , ${ }^{1}$ Gabriela Gomez, ${ }^{2}$ Nichola Kitson, ${ }^{1}$ Animesh Sinha (D) , ${ }^{3}$ \\ Natalia Yatskevich, ${ }^{4}$ Suzanne Staples, ${ }^{5}$ Ronelle Moodliar, ${ }^{5}$ Sharon Motlhako, ${ }^{6}$ \\ Matshepo Maloma, ${ }^{7}$ Mohammed Rassool, ${ }^{6}$ Nosipho Ngubane, ${ }^{7}$ Ella Ndlovu, ${ }^{7}$ \\ Bern-Thomas Nyang'wa ${ }^{8}$
}

To cite: Sweeney S, Gomez G, Kitson N, et al. Costeffectiveness of new MDR-TB regimens: study protocol for the TB-PRACTECAL economic evaluation substudy. BMJ Open 2020;10:e036599. doi:10.1136/ bmjopen-2019-036599

- Prepublication history for this paper is available online. To view these files, please visit the journal online (http://dx.doi. org/10.1136/bmjopen-2019036599).

Received 20 December 2019 Revised 26 August 2020 Accepted 07 September 2020

Check for updates

(c) Author(s) (or their employer(s)) 2020. Re-use permitted under CC BY-NC. No commercial re-use. See rights and permissions. Published by BMJ.

For numbered affiliations see end of article.

Correspondence to

Dr Sedona Sweeney;

sedona.sweeney@Ishtm.ac.uk

\section{ABSTRACT}

Introduction Current treatment regimens for multidrugresistant tuberculosis (MDR-TB) are long, poorly tolerated and have poor outcomes. Furthermore, the costs of treating MDR-TB are much greater than those for treating drug-susceptible TB, both for health service and patientincurred costs. Urgent action is needed to identify short, effective, tolerable and cheaper treatments for people with both quinolone-susceptible and quinolone-resistant MDRTB. We present the protocol for an economic evaluation (PRACTECAL-EE substudy) alongside an ongoing clinical trial (TB-PRACTECAL) aiming to assess the costs to patients and providers of new regimens, as well as their cost-effectiveness and impact on participant poverty levels. This substudy is based on data from the three countries participating in the main trial.

Methods and analysis Primary cost data will be collected from the provider and patient perspectives, following economic best practice. We will estimate the probability that new MDR-TB regimens containing bedaquiline, pretomanid and linezolid are cost-effective from a societal perspective as compared with the standard of care for MDR-TB patients in Uzbekistan, South Africa and Belarus. Analysis uses a Markov model populated with primary cost and outcome data collected at each study site. We will also estimate the impact of new regimens on prevalence of catastrophic patient costs due to TB.

Ethics and dissemination Ethical approval has been obtained from the London School of Hygiene \& Tropical Medicine and Médecins Sans Frontières. Local ethical approval will be sought in each study site. The results of the economic evaluation will be shared with the country health authorities and published in a peer-reviewed journal.

Trial registration number ClinicalTrials.gov Registry (NCT04207112); Pre-results.

\section{INTRODUCTION}

Multidrug-resistant tuberculosis (MDR-TB), defined as TB resistant to at least isoniazid and rifampicin, is an urgent public health issue. The current treatment regimen for

\section{Strengths and limitations of this study}

- This study will evaluate the cost-effectiveness and poverty impact of new regimens for multidrugresistant tuberculosis (MDR-TB) using prospectively collected primary data and modelling methods.

- Analyses will inform decision-making at national and global levels, while meeting the urgent need for evidence on improved drug regimens for MDR-TB.

- This will be one of the first cost-effectiveness studies on 6-month-long all-oral regimens for MDR-TB alongside a randomised controlled trial.

- The study is taking place in countries with a high prevalence of anti-TB drug resistance as well as a country with a high rate of HIV coinfection, covering some of the most challenging scenarios for MDR-TB treatment.

- Depending on recruitment progress at each trial site, sample sizes for patient costings may be restricted.

MDR-TB has poor outcomes and costs of treatment are greater than those for treatment of drug-susceptible TB, both in terms of costs to the health service as well as patientincurred costs. ${ }^{12}$ Key changes to recommendations for MDR-TB treatment regimens were published recently by the WHO after an assessment of new evidence. ${ }^{3}$ In this rapid communication, three main changes to the standard MDR regimen were made: first, the withdrawal of injectable antibiotics; second, the inclusion of bedaquiline in a recommended longer regimen (ie, 20 months) and third, the recommendation of use for a shorter regimen only for specific conditions. It also highlights the urgent need for evidence to inform better optimal treatment choices for MDR-TB patients. Economic evaluations of such bedaquiline-containing regimens will provide additional important 


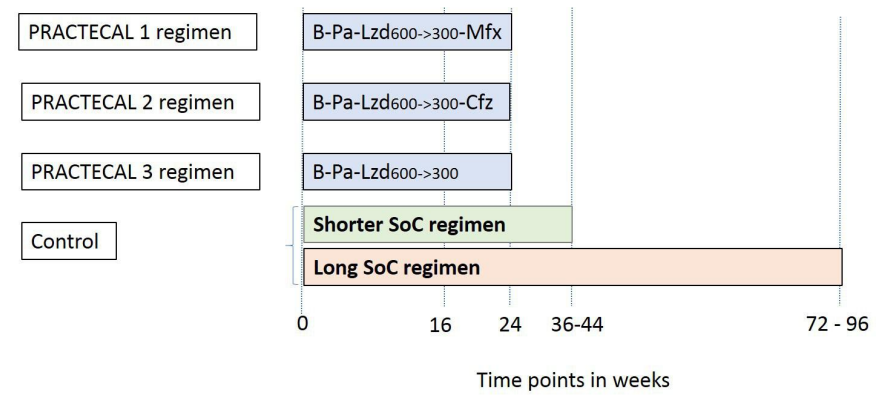

Figure 1 TB-PRACTECAL intervention and control drug regimens. B, bedaquiline (400mg daily for 2 weeks then 200mg three times per week for 22 weeks); Cfz, Clofazimine (50mg (less than $33 \mathrm{~kg}$ ), $100 \mathrm{mg}$ (more than $33 \mathrm{~kg}$ ) for 24 weeks); Mfx, moxifloxacin(400 mg once daily for 24 weeks); $\mathrm{Pa}$, pretomanid (200mg daily for 24 weeks); Lzd, linezolid (600mg for 16 weeks and then reduced to $300 \mathrm{mg}$ per day), SoC, Standard of care regimen locally approved and as much as possible conforming to the $\mathrm{WHO}$ recommendations for treatment of M/XDR-TB.

information for decision makers who need to consider its economic value along with clinical efficacy when planning for introduction.

TB-PRACTECAL is a randomised controlled trial to evaluate the safety and efficacy of investigational regimens containing bedaquiline and pretomanid for the treatment of MDR-TB (see figure 1) (ClinicalTrials.gov study registration NCT02589782). It has been designed in two stages; stage 1 is a phase II trial aiming to identify two regimens containing bedaquiline and pretomanid for further evaluation based on safety and efficacy outcomes after 8 weeks of treatment. Stage 2 is a phase III trial to evaluate the safety and efficacy of the two investigational regimens containing bedaquiline and pretomanid, selected in stage 1 , compared with the standard of care (SoC) at 72 weeks post randomisation. We present the protocol for an economic evaluation (PRACTECAL-EE substudy), taking place alongside the TB-PRACTECAL trial. This substudy aims to assess the costs to patients and providers of such regimens, and to estimate the costeffectiveness and impact on poverty levels for participants of introduction of new MDR-TB regimens in the three countries participating in the main trial.

Our decision problem is stated as the economic evaluation of the new treatment regimen for both quinolonesusceptible and quinolone-resistant MDR-TB patients, in order to inform evidence synthesis to Grading of Recommendations Assessment, Development, and Evaluation (GRADE) standards at a global level, and health technology assessments (HTA) in the trial host countries, as applied to regimens for MDR-TB. For both national and global assessments, the review of economic evidence produced alongside clinical trials focuses around patient outcomes and then on resources needed to answer the question of whether a new regimen should be considered for introduction. Population level and equity considerations can also be included, especially during the second stage, where the decision problem has advanced from whether to recommend a new regimen to how to introduce it to achieve maximum health outcomes with limited budgets.

\section{OBJECTIVES}

The overall aim of this substudy is to estimate the probability that 6-month all-oral MDR-TB regimens containing bedaquiline and pretomanid will be cost-effective from a societal perspective as compared with the SoC for MDR-TB patients in three settings: Uzbekistan, South Africa and Belarus.

A secondary aim is to assess the costs from a provider perspective of treating patients with these new regimens and estimate the impact of new regimens on prevalence of catastrophic costs due to TB.

The specific objectives of this substudy are, in each setting:

1. To assess the costs from a provider's perspective for selected facilities in the intervention and control arms.

2. To assess the costs from a patient's perspective for a sample of patients seeking care in study facilities in the intervention and control arms.

3. To estimate the prevalence of catastrophic costs in the intervention and control arms.

4. To assess the probability of new regimens being costeffective at different willingness-to-pay thresholds from a societal perspective using a Markov model.

\section{METHODS AND ANALYSIS \\ Study design}

This substudy is a cost-effectiveness analysis alongside a clinical trial. We will evaluate the total costs and effects of the study regimen (intervention) against the locally used WHO-approved regimens for treatment of multidrugresistant or extensively drug-resistant TB (M/XDR-TB) (control) using a Markov model populated with primary cost and outcome data collected at each study site.

This substudy will take place in three settings: Uzbekistan, South Africa and Belarus. The selection of countries and facilities in each country is based on their participation in the TB-PRACTECAL trial. In Uzbekistan, the trial is taking place in Tashkent city and six rayons (districts) in Karakalpakstan, in Western Uzbekistan (Nukus city, Takhiatash, Chimbay, Kegeily and Xodjeli rayons) implemented by the Republican Specialised ScientificPractical Medical Centre for Phthisiology and Pulmonology of the Republic of Uzbekistan. In South Africa, the trial is being conducted in Doris Goodwin and Don McKenzie Hospitals through the Tuberculosis and HIV Investigative Network, and Helen Joseph and King DinuZulu Hospitals though the Clinical HIV Research Unit at the University of the Witwatersrand. In Belarus, the trial is taking place in Minsk city and Oblast, implemented by the Republican Scientific and Practical Centre for Pulmonology and Tuberculosis of the Republic of Belarus. The trial sites were selected based on their likely ability 
to recruit at least 50 patients into the trial, together with the capacity to safely manage complex M/XDR-TB patients.

Our population of interest will be adults with Mycobacterium tuberculosis resistant to at least isoniazid and rifampicin by either molecular or phenotypic drug susceptibility testing. The sample size for the overall trial is 630. Patient enrolment in TB-PRACTECAL is currently underway, and cost data collection is anticipated to start in October 2020. The distribution of patient recruitment by country in the TB-PRACTECAL trial is expected to be approximately 280 patients in South Africa, 100 patients in Belarus and 250 patients in Uzbekistan. For cost data collection from the patient perspective, the numbers recruited into PRACTECAL-EE will be proportional to the overall trial, depending on the starting dates and numbers consenting. The level of patient costs and the expected change as a result of the intervention is unknown, therefore we have not done a sample size calculation for the patient costing survey. However, a sample size between 100 and 350 is comparable to previous patient costing studies from South Africa, Bangladesh, Indonesia, Tanzania and Brazil, which were found to give reasonable estimates with acceptable uncertainty. ${ }^{4-8}$ Data collection for costings from the provider perspective will be done at all trial sites in each country. Cost data collection is expected to be completed before the end of the main trial in 2021.

The perspective of the cost-effectiveness analysis will be societal, following requirements for the WHO guideline development process and best practice. ${ }^{9} 10$ The societal perspective will include all costs associated with delivery of care, regardless of whether incurred by providers or by patients and their households. The cost-effectiveness analysis will not include any costs or savings associated with informal support networks in the community, other caregivers or production costs at a societal level.

We will analyse and present costs incurred by the health system separately to inform future health system budgeting and allocation. We will also estimate the prevalence of catastrophic costs due to TB encountered at the household level in each trial arm. This analysis will help to inform whether the intervention facilitates progress towards the end TB target of 'zero catastrophic costs due to TB by $2020{ }^{\prime}{ }^{11}$

The model-based economic evaluation will focus on the comparison of two regimens selected for complete follow-up with SoC. The local SoC MDR-TB regimen will be used as the main comparator in the cost-effectiveness analysis. This corresponds to the control arm in the TB-PRACTECAL trial. This SoC arm will simulate the cohort following the most recent recommended regimen in each country.

The time frame for the cost-effectiveness analysis will be the time between the start of treatment and death of the entire simulated cohort in the Markov model.

\section{Data collection}

Data will be collected from various sources (trial, literature, national databases, questionnaires). Four types of data will need to be collected: (1) outcome data; (2) event and service use data; (3) cost data related to TB and (4) cost data unrelated to TB.

\section{Outcome data}

Event data from the trial for both intervention and control arms (for each patient type, age category, sex) will be collected on: number, reason (including but not limited to serious adverse events) and length of stay in hospital in each 24-week period over 108 weeks; mean number of outpatient visits per month in each 24-week period by reason over 108 weeks; mean number of monitoring tests in each 24-week period by reason over 108 weeks; duration and outcomes for serious adverse events, focusing on QT prolongation, indication of hepatotoxicity, hearing loss or nephrotoxicity and any service used to diagnose and treat adverse events as above. We will review the published literature (including data reported to $\mathrm{WHO}$ ) on probabilities of relapse and life expectancy in each setting to calculate disability-adjusted life years (DALYs) averted by the intervention.

\section{Cost data}

\section{Provider perspective}

Primary data collection from the provider perspective will gather information on the resources used to provide TB care under the new regimens as compared with the SoC. We will collect the economic, real-world, full costs of provision of MDR-TB care in intervention and control arms, from each setting alongside the TB-PRACTECAL trial following best-practice costing methods. ${ }^{12}$ We will employ standardised tools and instruments developed in the VALUE-TB study and in line with the Global Health Cost Consortium Reference Case for Estimating the Costs of Global Health Services and Interventions. ${ }^{12}$ Data collection will involve interviews with healthcare workers, retrospective review of project and expenditure records, physical measurement of building space and observation of service delivery. An ingredients approach will be taken, where the value of inputs is based on quantities and unit prices, including staff salaries, building space, training, supplies, drugs, equipment and overheads.

Costs related to adverse events will be estimated from the literature; if not available, we will then estimate the costs using an ingredient approach based on treatment protocols. We will aim to exclude costs related to trial only activities; however, data collection at trial sites may produce biased observations in terms of non-standard service delivery. We will aim to account for this in the uncertainty analysis of the cost effectiveness results. For this analysis, it may be necessary to interview national programme-level staff or access national salary scales and other information to understand the likely cost if new regimens were to be provided through the public sector and scaled up to the national level. 


\section{Patient perspective}

From a patient's perspective, we will collect self-reported data on patient-incurred costs related to accessing care in the intervention and control arms through a survey of patients enrolled in the TB-PRACTECAL trial (patient costing questionnaires are available for download at: https://doi.org/10.17037/DATA.00001799). Costs related to accessing the standard TB services will then be estimated from the information collected in the control arm. Information on costs encountered by TB patients and their households will be collected. Patient and household costs will include direct and indirect costs incurred because of their TB diagnosis. Direct costs will be defined as out-of-pocket medical (including consultation fees and any out-of-pocket payment for medicines and diagnostics) and non-medical expenses (including travel costs of participants and guardians, food costs incurred while in hospital, money spent buying any special foods or dietary supplements due to illness and any interest incurred on loans taken out to meet the costs of out-of-pocket payments). Cost will be estimated for the participant's local clinic (our study clinic), other public facilities, general practitioners, hospitals, traditional healers and pharmacies. Indirect costs will be estimated as the opportunity cost of time spent seeking care, plus any lost productivity due to illness.

Questionnaires will include detailed questions on care seeking behaviour and the above-mentioned cost variables. Questionnaires will also capture information about strategies adopted by participants to cope with costs of illness, including: taking interest-bearing loans from lenders, borrowing money from friends or relatives, selling personal goods and receipt of grants or charitable donations. Patient costs will be estimated for the entire episode of TB illness, from symptom onset to completion of treatment. Patient/household cost data will be collected retrospectively during visits aligned with other substudies, at baseline and weeks 24 and 48 for all patients. For those patients already enrolled that are answering their first patient cost questionnaire in week 24, we will also administer sections 1 and 2 of the baseline questionnaire, and costs prior to week 12 will be estimated based on the average cost per visit.

Collection of patient cost data within an economic evaluation of a clinical trial can be challenging and requires careful trade-off between available methodological approaches. ${ }^{13}$ As cost data collection for this substudy will be nested within the main trial, our approach to collecting cost data aims to limit survey fatigue wherever possible; for example, we use a recall-based survey rather than a cost diary. This is in line with existing methods for trial-based economic evaluation, but may result in underestimation of household costs due to potential telescoping and recall biases. Demographic data will be obtained from the main trial datasets, including gender, age, ethnicity and nationality and the impact of illness on normal productive patterns.

\section{Analysis}

\section{Cost analysis}

A descriptive analysis will be included in the patient cost analysis. We will use $\chi^{2}$ tests to determine the level to which the substudy sample is a representative of the sample in the main study for selected demographics.

Provider costs related to TB care will be estimated as one-time costs required for introduction and recurrent costs required for sustaining the intervention. Costs will be analysed as total costs, unit costs and incremental costs disaggregated by input categories (ie, staff, capital, drugs). Any costs measured from a provider or patient perspective necessary only for research purposes (such as nurse or patient time allocated to administration of patient consent) will be excluded from the analysis.

Patient costs will be analysed in total and by subcategory, including direct medical costs (drugs, diagnostics, etc), direct non-medical costs. (eg, transport, special food or supplements), productivity loss associated with ill health (inability to work or reduced productivity while at work) and/or time costs associated with seeking care (eg, travel and consultation time for health facility visits). Patient costs data will be included as part of societal costs for the cost-effectiveness analysis. In addition, a separate analysis will be conducted to estimate the prevalence of catastrophic costs in intervention and control populations.

The main trial is expected to report on its main outcomes in 2021; all cost information collected in the previous year will be inflated to 2021 currency and converted from local currency units to US dollars and purchasing-power parity using exchange rates for that year.

\section{Cost-effectiveness analysis}

Based on the requirements of the GRADE process for global-level evaluations, we will develop a Markov model to follow a cohort of MDR-TB patients under the different treatment options. A Markov model is appropriate for estimating the expected effects of the intervention at patient level, including: (1) improved treatment efficacy (better cure rate); (2) improved safety profile (fewer adverse events) and (3) shorter regimen (higher completion rate). The treatment effects on transmission of MDR-TB will not be modelled.

We will estimate incremental costs, cost per death averted and cost per DALY averted of the intervention(s) to the comparator. Discount rates of $3 \%$ will be used to discount both costs and effects in the primary analysis, following international reference case recommendations. ${ }^{9}{ }^{12}$ We will vary these rates in sensitivity analyses. We will explore the effects of sampling uncertainty for the estimated incremental cost and incremental effectiveness parameters, together with the impact of methodological assumptions (such as discount rate, study perspective) using deterministic and probabilistic sensitivity analyses. Where applicable, we will report differences in costs, outcomes or cost-effectiveness that can be explained by variations between subgroups of patients with different baseline characteristics. Results will be presented using 
cost-effectiveness acceptability curves for a range of willingness to pay thresholds.

The main outputs from the model will include:

- Incremental cost incurred per DALY averted with the intervention regimen, compared with the SoC from a societal perspective.

- Incremental cost per DALY averted with the intervention regimen, compared with the SoC from a provider perspective.

Secondary outcome measures from the model will include:

- The mean cost per month of treatment for different regimens and type of patient (MDR-TB, pre-XDR-TB (resistant to fluoroquinolone) and XDR-TB).

- The mean cost per course of treatment for different types of patients (MDR-TB, pre-XDR-TB (resistant to fluoroquinolone), XDR-TB) and by category (training, monitoring, service delivery and drugs).

- The incremental total cost of intervention for the trial population, over the trial duration.

- The incremental total cost of intervention for the modelling cohort, over a lifetime horizon.

The model will be parameterised using primary data from the TB-PRACTECAL trial on effectiveness (outcomes post 108 weeks of trial follow-up) and costs, combined with secondary data on national population characteristics and outcomes with the current SoC. Expert opinion will be used where no data are available, for example, estimating the extent to which patients return to care after default. All model parameters will be reviewed by a panel of local experts.

We will perform additional analyses disaggregated by patient type, sex, age and socioeconomic status, and the main determinants of cost-effectiveness, using a sensitivity analysis to inform decisions for other settings. We will also conduct a sensitivity analysis with intermediate durations, as cost estimates based on study subjects' lifetimes can be marked by very large confidence intervals.

\section{ETHICS AND DISSEMINATION}

Ethical approval has been obtained from the London School of Hygiene \& Tropical Medicine (reference 17580) and Médecins Sans Frontières (reference 1541c). Local ethical approval is currently being sought from relevant agencies in each study setting. In South Africa, this includes PharmaEthics and University of the Witwatersrand Human Research's ethics committee. In Uzbekistan, this is the ethical committee under Ministry of Health of the Republic of Uzbekistan. In Belarus, this is the ethics committee of the State Institution Republican Scientific and Practical Centre of Pulmonology and Tuberculosis, and the Centre of Expertise for Testing in Healthcare. For the provider costings, staff will be informed of the study in their language of preference and will be asked to give written informed consent before participating in the study. Approval from the hospital management at each site will be sought and obtained before starting the provider cost data collection. We will seek written informed consent of patients before conducting the patient costing questionnaire.

Following the end of the research project and publication of findings, cost data will be made available through an approved and curated open-access platform. All data will be fully anonymised before being placed in the public domain.

The results of the economic evaluation will be published in a peer-reviewed journal. If the TB-PRACTECAL trial successfully identifies effective and safe regimens, the results of this study will inform a GRADE process by potentially answering a specific population, intervention, comparison, and outcomes (PICO) question on costeffectiveness of the PRACTECAL regimens, as applied by the WHO guidelines committee processes. The results may also be used for HTA in the trial host countries.

\section{Patient and public involvement}

Patients and, where feasible, the community have been engaged in the set-up and implementation stages of the main TB-PRACTECAL trial. ${ }^{14} 15$ Prior to rollout of the PRACTECAL-EE questionnaires, we will conduct consultations with patients and/or health workers in each setting to gain a sense of the types of costs encountered by TB patients and how best to approach patients to engage in a conversation about resources before rolling out costing data collection.

\section{DISCUSSION}

The novel regimens in the TB-PRACTECAL trial could potentially improve the patient experience by shortening treatment duration, reducing pill burden and reducing adverse events. If the study regimens are proven noninferior to the standard of care, evidence on the costeffectiveness of these regimens will be critical to help policy makers evaluate the economic impact of adopting these new regimens. The PRACTECAL-EE substudy will provide evidence from both the patients' and health systems' perspective to assist in policy development following publication of outcomes from the clinical trial.

\section{Author affiliations \\ ${ }^{1}$ Global Health and Development, London School of Hygiene and Tropical Medicine, London, UK \\ ${ }^{2}$ Vaccine Epidemiology and Modelling, Sanofi Pasteur SA, Lyon, France \\ ${ }^{3}$ Médecins Sans Frontières Holland, Minsk, Belarus \\ ${ }^{4}$ Republican Scientific and Practical Centre for Pulmonology and Tuberculosis, \\ Minsk, Belarus \\ ${ }^{5}$ TB and HIV Investigative Network (THINK), Durban, South Africa \\ ${ }^{6}$ Helen Joseph Hospital, Clinical HIV Research Unit, Wits Health Consortium, University of the Witwatersrand, Johannesburg, South Africa \\ ${ }^{7}$ King DinuZulu Hospital, Clinical HIV Research Unit, Wits Health Consortium, \\ University of the Witwatersrand, Durban, South Africa \\ ${ }^{8}$ Manson Unit, Médecins Sans Frontières, London, UK}

Twitter Animesh Sinha @animesh4767 and Bern-Thomas Nyang'wa @docwak77

Acknowledgements The authors would like to thank the following individuals who provided a peer review of a draft of this protocol: Nick Menzies, Lorna Guinness and 
David Dowdy. Emma Veitch, freelance medical editor for MSF, UK, provided editorial assistance and her work was funded by MSF UK.

Contributors SS and GBG outlined the original design of this economic substudy and drafted the protocol. NK, AS, NY, SS, RM, SM, MM, MR, NN and EN commented on working drafts and approved the final draft of the protocol. B-TN contributed to the drafting of the protocol and is the guarantor of the overall TB-PRACTECAL study. SS is the guarantor of the PRACTECAL-EE substudy. All authors commented and approved the final draft of the protocol.

Funding The primary funder of the trial is Médecins sans Frontières.

Competing interests The chief investigator of the TB-PRACTECAL trial (B-TN) is a full-time employee of Médecins sans Frontières, the funder of the research. GBG is currently employed by Sanofi Pasteur as Regional Lead for vaccine epidemiology and modelling on Europe and does not work in any project related to tuberculosis. GBG's contribution to this work pertains to activities prior to employment at Sanofi Pasteur.

Patient and public involvement Patients and/or the public were involved in the design, or conduct, or reporting, or dissemination plans of this research. Refer to the Methods section for further details.

Patient consent for publication Not required.

Provenance and peer review Not commissioned; externally peer reviewed.

Open access This is an open access article distributed in accordance with the Creative Commons Attribution Non Commercial (CC BY-NC 4.0) license, which permits others to distribute, remix, adapt, build upon this work non-commercially, and license their derivative works on different terms, provided the original work is properly cited, appropriate credit is given, any changes made indicated, and the use is non-commercial. See: http://creativecommons.org/licenses/by-nc/4.0/.

ORCID iDs

Sedona Sweeney http://orcid.org/0000-0003-4233-9080

Animesh Sinha http://orcid.org/0000-0003-3213-7813

\section{REFERENCES}

1 Laurence YV, Griffiths UK, Vassall A. Costs to health services and the patient of treating tuberculosis: a systematic literature review. Pharmacoeconomics 2015;33:939-55.

2 Collaborative Group for the Meta-Analysis of Individual Patient Data in MDR-TB treatment-2017, Ahmad N, Ahuja SD, et al. Treatment correlates of successful outcomes in pulmonary multidrug-resistant tuberculosis: an individual patient data meta-analysis. Lancet 2018;392:821-34.

3 WHO. Rapid communication: key changes to treatment of multidrugand rifampicin-resistant tuberculosis (MDR/RR-TB). Available: http://www.who.int/tb/publications/2018/WHO_RapidCommunicati onMDRTB.pdf?ua $=1$

4 Gospodarevskaya E, Tulloch O, Bunga C, et al. Patient costs during tuberculosis treatment in Bangladesh and Tanzania: the potential of shorter regimens. Int J Tuberc Lung Dis 2014;18:810-7.

5 Foster N, Vassall A, Cleary S, et al. The economic burden of TB diagnosis and treatment in South Africa. Soc Sci Med 2015;130:42-50.

6 Ramma L, Cox H, Wilkinson L, et al. Patients' costs associated with seeking and accessing treatment for drug-resistant tuberculosis in South Africa. Int J Tuberc Lung Dis 2015;19:1513-9.

7 Trajman A, Bastos ML, Belo M, et al. Shortened first-line TB treatment in Brazil: potential cost savings for patients and health services. BMC Health Serv Res 2016;16:27.

8 Fuady A, Houweling TAJ, Mansyur M, et al. Catastrophic total costs in tuberculosis-affected households and their determinants since Indonesia's implementation of universal health coverage. Infect Dis Poverty 2018;7:1-14.

9 Wilkinson T, Sculpher MJ, Claxton K, et al. The International decision support initiative reference case for economic evaluation: an aid to thought. Value Health 2016;19:921-8.

10 Sanders GD, Neumann PJ, Basu A, et al. Recommendations for conduct, methodological practices, and reporting of costeffectiveness analyses: second panel on cost-effectiveness in health and medicine. JAMA 2016;316:1093-103.

11 World Health Organization. The end TB strategy. Geneva, Switzerland, 2015.

12 Vassall A, Sweeney S, Kahn JGJ, et al. Reference case for estimating the costs of global health services and interventions. global health cost Consortium, 2017. Available: https://ghcosting.org/pages/ standards/reference_case

13 Sweeney S, Vassall A, Foster N, et al. Methodological issues to consider when collecting data to estimate poverty impact in economic evaluations in low-income and middle-income countries. Health Econ 2016;25(Suppl 1):42-52.

14 The BMJ. Engaging communities in tuberculosis research: the experience of the TB-PRACTECAL trial. Available: https://blogs.bmj. $\mathrm{com} / \mathrm{bmj} / 2018 / 11 / 09 /$ engaging-communities-tuberculosis-researchexperience-practecal-trial/ [Accessed 16 Dec 2019].

15 Babaniyazov A, Nyang'wa BT, Pardington N. 'Trial and Error' operational reflections on the set-up of a community engagement programme for a phase ii/iii clinical trial to identify new MDR-TB treatments in Uzbekistan. In: Médecins sans Frontières scientific day South Asia (not peer reviewed), 2016. 